\title{
Familial aggregation of tonsillectomy in early childhood and adolescence
}

\author{
Peter Bager \\ Giulia Corn \\ Jan Wohlfahrt \\ Heather A Boyd \\ Bjarke Feenstra \\ Mads Melbye
}

Department of Epidemiology Research, Statens Serum Institut,

Copenhagen, Denmark
Correspondence: Peter Bager

Department of Epidemiology Research, Statens Serum Institut, Artillerivej 5/

Ørestads boulevard 5, 2300 Copenhagen, Denmark

Tel +4532688327

Email pbg@ssi.dk
This article was published in the following Dove Press journal: Clinical Epidemiology

Background: The tonsils are immunological gatekeepers against pathogens. Immunological response to tonsillitis may vary clinically from no enlargement of the tonsils to nearly obstructive conditions. In this investigation, we studied the familial aggregation of tonsillectomy, as an indicator of the extent to which tonsillar immune responses to infections might be genetically controlled. Methods: Data on kinship relations and vital status from the Danish Civil Registration System were used to establish a cohort of Danes with relatives born since 1977. Tonsillectomies in all hospitals and clinics from 1977 to 2013 were identified in national registers together with the indication for tonsillectomy. Rate ratios (RRs) for tonsillectomy $>1$ year after tonsillectomy in specific types of relatives (first to fourth degree) were estimated in Poisson regression models with adjustment for calendar period, sex, age, and total number of specified relatives.

Results: A cohort of 2.4 million persons was followed for 44,100,697 million person-years (mean 18.4 years/person), and included 148,190 tonsillectomies. RRs of tonsillectomy were consistently higher when the relatedness and the number of tonsillectomized relatives were higher. RRs were similar in boys and girls, but were larger in early childhood. Additional analyses suggested that this relatively higher RR at younger ages was due to a larger influence of shared environment at younger ages, whereas the genetic influence was similar at all ages. Results were similar for tonsillectomies performed strictly due to tonsillitis.

Conclusions: Genetic factors appear to predispose to severe tonsillitis underlying tonsillectomies, regardless of age and sex. Further studies are needed to understand how genes regulate the tonsils' immune response against infections.

Keywords: tonsillitis, epidemiology, hereditary, risk factors, infection

\section{Introduction}

The tonsils are the immunological gatekeepers against pathogens entering through the mouth and nose, as their specialized crypt structure effectively captures antigens. ${ }^{1}$ Subsequent immune responses may result in tonsillitis and tonsil enlargement of varying severity, ranging from temporary narrowing of the pharynx with accompanying tenderness to dysphagia, dyspnea, and often severe bouts of tonsillitis. Tonsillectomy is considered to reduce obstructive symptoms, the frequency of tonsillitis episodes, and the number of infections beginning in the tonsils and spreading elsewhere in the respiratory tract. ${ }^{2-4}$

In Denmark, $6 \%-9 \%$ of persons $<20$ years of age underwent tonsillectomy in the period between 1980 and 2001. ${ }^{5}$ However, tonsillectomy rates vary greatly during childhood, ${ }^{6-9}$ with peaks in early childhood (age 4-5 years) and adolescence (age 16-17 years).$^{5,8,9}$ Admissions for chronic tonsillar disease (with or without accompanying 
tonsillectomy) demonstrate similar patterns. ${ }^{5}$ The reason for these peaks remains unclear, but both environmental and genetic factors may play a role. Immune responses to infections may be attributed to a degree of genetic control, for example, as indicated by the role of the CCR5 gene in controlling time to acquired immunodeficiency syndrome and the role of the mannose-binding lectin gene in controlling effective responses to infant infections. ${ }^{10,11}$ In the case of tonsillitis episodes, twin studies indicate a considerable genetic component underlying recurrent tonsillitis and tonsillectomy. ${ }^{12,13}$ For example, 1 twin study reported age at tonsillectomy to be more similar among monozygotic than dizygotic twins. ${ }^{13}$

We studied the potential influence of genetic control of the tonsillar immune response by using Denmark's national population-based registers and the Danish Family Relations Database to describe the familial aggregation of nearly 150,000 tonsillectomies by age and sex. After considering the role of environmental factors, ${ }^{5,13}$ such as familial preferences for tonsillectomy, we modeled the familial aggregation to isolate a possible genetic component using principles of basic heredity patterns.

\section{Methods}

\section{Data sources}

\section{Civil registration system}

Using the unique personal identification number assigned to each Danish resident, the Civil Registration System has since 1968 registered demographic and vital status information of all persons residing in Denmark. ${ }^{14,15}$ The personal identification number permits follow-up of all persons living in Denmark and accurate linking of individual-level information from Denmark's nationwide population-based registers.

\section{National hospital discharge register}

The hospital discharge register contains discharge diagnoses of all patients admitted to Danish hospitals since 1977, hospital outpatient diagnoses from 1995 onward, and procedure codes for all surgical procedures performed in Danish hospitals since $1977 .{ }^{16}$ Between 1977 and 1995, surgical procedures were coded using the Danish National Board of Health's Classification of Surgical Procedures and Treatments (OBK, first to third eds.). Since 1996, surgical procedures have been coded using the Nordic Medico-Statistical Committee Classification of Surgical Procedures (NCSP). Between 1977 and 1993, discharge diagnoses were coded using the International Classification of Diseases (ICD), version 8, with version 10 being used since 1994.

\section{National health service register}

The health service register has records of all primary health care services delivered to persons in Denmark since 1990, including the provider's medical specialty, the type of service, and the calendar week in which the service was provided. ${ }^{17,18}$ The register is virtually complete because registration is required for providers to be reimbursed by the state, and the state reimburses for most services (universal healthcare). In recent decades, many planned inpatient tonsillectomies have become outpatient procedures, being performed either in hospital settings or in otolaryngology clinics. ${ }^{19}$

\section{Danish family relations database}

The database is based on kinship information in the Civil Registration System and permits the identification of relatives for persons with family in Denmark. ${ }^{20-24}$ Parents, siblings and half-siblings can be identified for nearly all persons born from 1950 and onwards (presently 2013), whereas grandparents, aunts/uncles and cousins are identifiable for almost $90 \%$ of persons born from 1985 and onwards (presently 2013).

\section{Ethics}

The study was based on existing data in national registries and was approved by the Danish Protection Agency (j.nr. 2015-57-0102). Because subjects were not contacted as part of the study, written informed consent was not required.

\section{Study population}

Using the Civil Registration System, we established a cohort of persons born in Denmark who were alive in 1977, or born later, and for whom any of the relatives were born in Denmark since 1977. In this way, cohort members had nearly complete registration of tonsillectomies and also constituted about half of the Danish population. Thus, the sample size of the cohort was determined by the size of the Danish population and there was no pre-study calculation of sample size. Cohort members were followed from January 1, 1977, or date of birth, whichever came last, until the first of the following events: 1) tonsillectomy (outcome of interest); 2) death; 3) emigration; 4) classified "missing" in the Civil Registration System; or 5) October 31, 2013 (end of follow-up). Also see Methods section in the Supplementary materials.

\section{Identification of tonsillectomies}

Persons registered in the National Hospital Discharge Register or the National Health Services Register during the follow-up period with any of the following codes were considered to have undergone tonsillectomy: OBK code 2614 (tonsillectomy); 
NCSP codes EMB10 (tonsillectomy) and EMB20 (adenotonsillectomy); and service code 3015 (tonsillectomy) reimbursed to otolaryngologists (medical specialty number 21 ). Information on indications was available for tonsillectomies performed in hospitals: tonsillitis was identified using ICD codes for acute, chronic, recurrent, or streptococcal tonsillitis (ICD8 codes 0340.0, 4630.x, 5000.0, 5000.2; ICD10 codes J03.X, J35.0). Overall, the most common diagnosis codes underlying the tonsillectomy indication were tonsillitis $(60 \%)$, tonsil hypertrophy (19\%), peritonsillar abscess (11\%), and vegetative adenoids (4\%); the remaining included more than 50 other diagnoses $(6 \%)$.

\section{Identification of relatives and determination of relatives' history of tonsillectomy}

For each cohort member, we used the Danish Family Relations Database to identify any relatives of the same generation (twins, siblings, half-siblings, cousins, halfcousins) or the previous generation (parents, uncles/aunts, half-uncles/half-aunts) registered in the Civil Registration System. Once all relatives were identified, we determined which, if any, underwent tonsillectomy before their respective cohort member underwent tonsillectomy (exposure of interest). Biological relatedness was defined by degrees and coefficients and was as follows: ${ }^{25}$ same sex twins were assumed to comprise 50\% monozygotes (first-degree relatives, coefficient 1.000) and 50\% dizygotes (first-degree relatives, coefficient 0.500 ), yielding a combined coefficient of $0.500 \times 1.000+0.500 \times 0.500=0.750$; different sex twins comprised $100 \%$ dizygotes (first-degree relatives, coefficient 0.500); siblings and parents (first-degree relatives, coefficient 0.500); half-siblings and uncles/aunts (seconddegree relatives, coefficient 0.250 ); cousins and half-uncles/ aunts (third-degree relatives, coefficient 0.125 ); half-cousins (fourth-degree relatives, coefficient 0.0625 ).

\section{Statistical analyses}

Familial aggregation of tonsillectomy was evaluated by comparing the rates of tonsillectomy in individuals with and without previously tonsillectomized relatives. More specifically, incidence rate ratios (RRs) were estimated comparing the rate of tonsillectomy for individuals with a given number $(1,2$, or $\geq 3)$ of relatives of a certain type who had previously undergone tonsillectomy with the rate for individuals with at least the same number of relatives of the given type, none of whom had undergone the procedure. For example, when evaluating the risk of tonsillectomy associated with 2 tonsillectomized siblings, the
RR compared the rate of tonsillectomy in individuals with 2 previously tonsillectomized siblings with that of individuals with at least 2 siblings, all of whom still retained their tonsils. We considered only previous tonsillectomies in relatives to ensure that when a pair of individuals were both cohort members, the pair contributed only once to our estimates.

RRs were estimated for each type of relative separately using log-linear Poisson regression models in SAS, version 9.2 (SAS Institute, Inc., Cary, NC, USA), with adjustment for calendar period (1-year categories) and number of relatives of a specified type, as well as for the interaction between sex and attained age (1-year categories).

In initial analyses, we evaluated the effect of the time elapsed between a relative's tonsillectomy and tonsillectomy in a cohort member; we found higher rates of tonsillectomy associated with very recent tonsillectomy in a relative ( $<1$ year previously) (Table S1), whereas rates associated with longer intervals were lower. Since shared sources of infections are most likely overrepresented shortly after "exposure", we only present results for tonsillectomies occurring $\geq 1$ year after a relative's tonsillectomy.

In analyses estimating the age-interval-specific RRs, we excluded tonsillectomies at age $20+$ years of both cohort persons and their relatives, because of small numbers. Furthermore, we presented the rate of tonsillectomy in a given age interval in individuals with and without a relative who were tonsillectomized in the same age interval, because the RR for comparisons with different age intervals were slightly lower (Table S2).

To evaluate the degree to which observed familial aggregation was due to shared genes vs shared environment, the RRs for family history were modeled as the product of a genetic component and a shared environmental component. In this context, we differentiated between RRs estimated for family history ("observed RRs"), and RRs estimated in the model ("estimated RRs"). For the technical details, see Methods section in the Supplementary materials.

\section{Results}

The study population included 2,399,717 individuals with at least one identifiable relative, and a total of 44,100,697 person-years of follow-up (mean: 18.4 years/person). Tonsillectomy was performed in 148,190 individuals from 1977 to 2013 , with $133,552(90.1 \%)$ performed in hospitals and $14,638(9.9 \%)$ in otolaryngology clinics. Adenotonsillectomies constituted $29.8 \%$ of tonsillectomies performed in hospitals since 1996.

Table 1 shows the RR for tonsillectomy by type and number of tonsillectomized relatives. The rate of tonsillectomy 
Table I Rate ratio for tonsillectomy by type and number of tonsillectomized relatives, Denmark, 1977-2013

\begin{tabular}{|c|c|c|c|}
\hline \multirow{2}{*}{$\begin{array}{l}\text { Type and number of } \\
\text { tonsillectomized relatives }\end{array}$} & \multicolumn{3}{|l|}{ Tonsillectomy } \\
\hline & Person-years $\times 10^{3}$ & Number of events & Rate ratio $(95 \% \mathrm{Cl})$ \\
\hline \multicolumn{4}{|l|}{ Twins } \\
\hline No & 662.40 & 2,037 & I (Reference) \\
\hline Yes (same-sex twin) & 14.84 & 158 & $4.05(3.44-4.76)$ \\
\hline No & 347.14 & $\mathrm{I}, 247$ & I (Reference) \\
\hline Yes (different-sex twin) & 10.59 & 102 & $3.01(2.46-3.69)$ \\
\hline \multicolumn{4}{|l|}{ First-degree relatives } \\
\hline \multicolumn{4}{|l|}{ Siblings } \\
\hline No & $29,050.40$ & 89,798 & I (Reference) \\
\hline Yes, I & I,329.72 & 10,532 & $3.12(3.05-3.18)$ \\
\hline Yes, 2 & 63.19 & 896 & $6.12(5.72-6.55)$ \\
\hline Yes, 3+ & 4.09 & 96 & $10.87(8.88-13.31)$ \\
\hline \multicolumn{4}{|l|}{ Parents } \\
\hline No & $\mathrm{I}, 500.65$ & 5,057 & I (Reference) \\
\hline Yes, I & 249.61 & 2,338 & $2.80(2.67-2.95)$ \\
\hline Yes, both & 8.56 & 177 & $6.76(5.79-7.88)$ \\
\hline \multicolumn{4}{|l|}{ Second-degree relatives } \\
\hline \multicolumn{4}{|l|}{ Maternal half-siblings } \\
\hline No & $5,001.56$ & 17,872 & I (Reference) \\
\hline Yes, I & 339.55 & 2,104 & $1.91(1.82-2.00)$ \\
\hline Yes, 2 & 19.48 & 199 & $3.23(2.80-3.72)$ \\
\hline Yes, 3+ & 1.13 & 22 & $6.26(4.10-9.56)$ \\
\hline \multicolumn{4}{|l|}{ Paternal half-siblings } \\
\hline No & $5,769.94$ & 19,393 & I (Reference) \\
\hline Yes, I & 408.58 & $\mathrm{I}, 794$ & $1.42(1.36-1.50)$ \\
\hline Yes, 2 & 27.97 & 169 & $1.98(1.70-2.31)$ \\
\hline Yes, 3+ & 1.82 & 9 & $1.53(0.79-2.94)$ \\
\hline \multicolumn{4}{|l|}{ Uncles/aunts } \\
\hline No & $3,330.29$ & $|2,77|$ & I (Reference) \\
\hline Yes, I & 397.61 & 2,432 & $1.62(1.55-1.70)$ \\
\hline Yes, 2 & 25.43 & 248 & $2.68(2.36-3.05)$ \\
\hline Yes, 3+ & 2.79 & 43 & $4.37(3.23-5.93)$ \\
\hline \multicolumn{4}{|l|}{ Third-degree relatives } \\
\hline \multicolumn{4}{|l|}{ Cousins } \\
\hline No & $20,658.13$ & 71,811 & I (Reference) \\
\hline Yes, I & $2,773.47$ & $1 \mathrm{I}, 682$ & $1.35(1.32-1.38)$ \\
\hline Yes, 2 & 636.72 & 3,118 & $1.64(1.58-1.70)$ \\
\hline Yes, 3+ & 187.74 & $\mathrm{I}, 054$ & $1.99(1.87-2.11)$ \\
\hline \multicolumn{4}{|l|}{ Half-uncles/aunts } \\
\hline No & $2,433.84$ & 10,333 & I (Reference) \\
\hline Yes, I & 326.35 & $\mathrm{I}, 792$ & $1.31(1.25-1.38)$ \\
\hline Yes, 2 & 31.25 & 233 & $1.77(1.55-2.02)$ \\
\hline Yes, 3+ & 3.64 & 26 & $1.67(1.13-2.47)$ \\
\hline \multicolumn{4}{|l|}{ Fourth-degree relatives } \\
\hline \multicolumn{4}{|l|}{ Half-cousins } \\
\hline No & $3,521.07$ & 14,776 & I (Reference) \\
\hline Yes, I & 427.06 & 1,914 & $1.11(1.06-1.16)$ \\
\hline Yes, 2 & 90.38 & 425 & $1.18(1.07-1.30)$ \\
\hline Yes, 3+ & 23.83 & 119 & $1.28(1.07-1.54)$ \\
\hline
\end{tabular}

Notes: All rate ratios are adjusted for calendar period, number of relatives of the specified type, and the interaction between attained age and sex. "a"No" means that although the person had relatives of the given type and number, none of these relatives had been tonsillectomized.

following tonsillectomy in relatives was up to 11 times higher (range 1.11-10.87) than the rate in persons whose relatives had not undergone the procedure. RR magnitudes increased with increasing degree of relatedness: having tonsillectomized siblings or parents yielded the greatest RRs, while the smallest increases in tonsillectomy rate were seen among persons with tonsillectomized cousins, half-cousins and half-uncles/ aunts (Table 1). RR magnitudes also increased with increasing 
numbers of tonsillectomized relatives of a given degree of relatedness (Table 1). For example, having 2 tonsillectomized siblings or parents was associated with a 6 -fold increase in the rate of tonsillectomy, and having 3 tonsillectomized siblings was associated with an almost 11-fold increase in the tonsillectomy rate. These findings were not materially different when stratified by total number of relatives (Table S3 $)$. We observed higher RRs of tonsillectomy when having tonsillectomized relatives on the maternal side than when having tonsillectomized relatives on the paternal side, although these patterns appeared limited to close maternal relatives (maternal half-siblings, Table 1), and were not evident in more distant relatives (e.g., maternal half-cousins, Table S4).

Figure $1 \mathrm{~A}$ and $\mathrm{B}$ shows observed RRs - illustrated by colored data points - for tonsillectomy at age $<10$ years and 10-19 years, respectively, according to coefficient of relatedness to tonsillectomized relatives. The RRs for each data point are shown in Table S5. Generally, RRs were higher for children $<10$ years of age (Figure $1 \mathrm{~A}$ ) than for older children (Figure 1B). For example, RRs for tonsillectomy following tonsillectomy in 1 sibling were 5.03 at $<10$ years of age and 2.42 at $10-19$ years. Furthermore, for children $<10$ years of age (Figure 1A), associations were generally stronger for tonsillectomy in relatives with the same mother (full siblings and maternal half-siblings, red data points) than for relatives with different mothers (blue data points) but the same level of genetic relatedness. Such a difference was not observed for children aged 10-19 years (Figure 1B).

Figure $1 \mathrm{~A}$ and $\mathrm{B}$ also include results from models of the genetic and environmental components of the familial aggregation of tonsillectomy in each of the 2 age groups. Figure $1 \mathrm{C}$ illustrates the key aspects of Figure 1A and B: the dashed line represents the genetic component, which increases with increasing degree of relatedness, while the red and blue lines represent the environmental component for relatives with the same mother and different mothers, respectively. Overall, the genetic component was estimated to be of similar size in the 2 age groups (as seen by the similar slopes of the dashed lines for the 2 age groups, also see the estimates in Methods section in the Supplementary materials), whereas
A

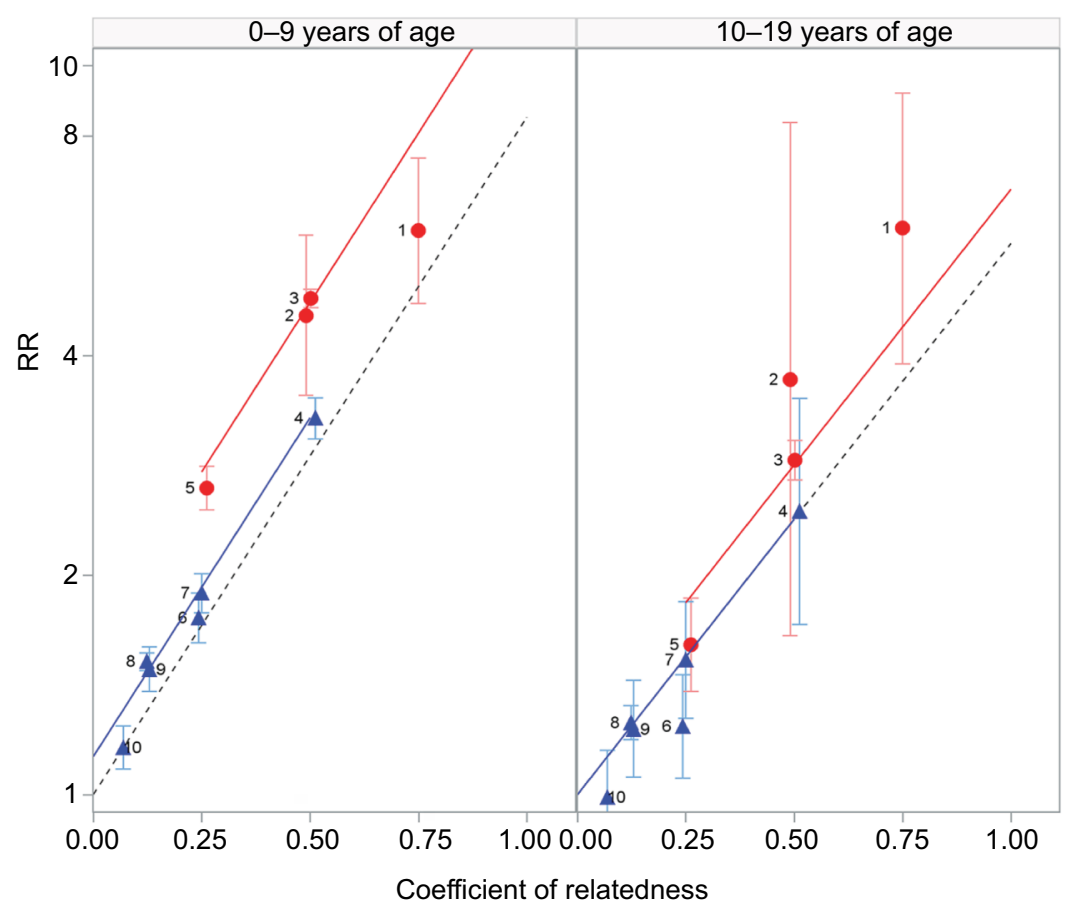

C

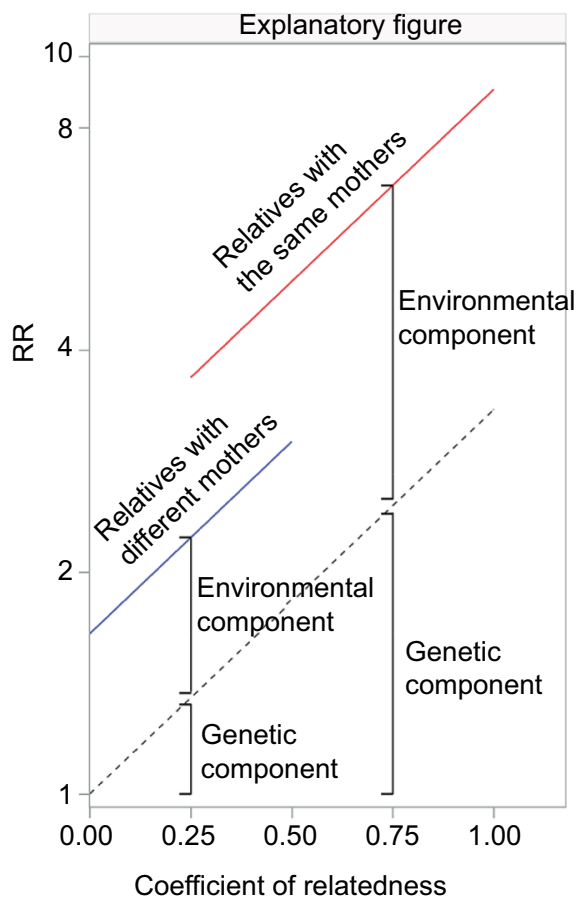

Figure I RRs for tonsillectomy at age <10 years (A) and 10-19 years (B) according to relatedness to tonsillectomized relatives, Denmark, I977-20I3. Notes: Relatedness is estimated by coefficients. The points (red circles and blue triangles) represent the "observed" RRs associated with having I tonsillectomized relative of a given type (Table S5). The lines represent corresponding RRs estimated by the statistical model (C). For the technical details, see Methods section in the Supplementary materials. Briefly, the genetic component for the 2 age groups is very similar, as seen by the very similar slopes of all lines, that is, 2.91 in (A) and 2.39 in (B) (see values in Table SI). In contrast, the environmental component was strongest in the young group for relatives with the same mother, as seen by the larger intercept of the red line in A ( 1.83 vs 1.19 for the blue line) than in B ( 1.13 vs 1.00 for the blue line) (see values in Table SI). Where Cls associated with different estimates overlapped, the position of the points and intervals was shifted slightly on the $\mathrm{X}$-axis for clarity of reading, and the part below I is not shown in I case $(0.85-\mathrm{I} .00)$. RR, $\Delta=$ different mother, $\bullet=$ same mother, I=same sex twin $(0.75), 2=$ different sex twin $(0.50), 3=$ sibling $(0.50), 4=$ parent $(0.50), 5=$ maternal half-sibling $(0.25), 6=$ paternal half-sibling $(0.25), 7=$ uncle/aunt $(0.25)$, $8=$ cousin $(0.125)$, $9=$ half-uncle/aunt $(0.125), 10=$ half-cousin $(0.06)$.

Abbreviation: $\mathrm{RR}$, rate ratio. 
a contribution from a shared environmental component was primarily observed in the youngest group (as seen by a larger intercept in the younger age group, also see the estimates in Methods section in the Supplementary materials). In fact, in the older group, the contribution from shared household (same mother) decreases considerably, while the non-shared household effect (different mothers) disappears.

Figure 2 shows observed and estimated RRs for tonsillectomy in children with a previously tonsillectomized sibling, by age, along with the estimated contributions from genetics (dark gray area) and the shared environment (light gray area). These results supported the notion from Figure $1 \mathrm{~A}$ and $\mathrm{B}$ that the particularly strong familial aggregation of tonsillectomy observed in younger children was due to a larger contribution of shared environment in this age group, whereas the genetic component of the association was similar for both age groups (for further background on this result, see Methods section in the Supplementary materials). Similar results for children with a tonsillectomized cousin are shown in Figure S1.

The age-specific pattern shown in Figure 2 was observed in both males and females (Figure S2A and $\underline{B}$ ). Furthermore, the RR for tonsillectomy associated with 1 sibling with tonsillectomy did not differ substantially by sex of the tonsillectomized sibling, for both males and females and among children $<10$ years and 10-19 years of age (Table S6).
When we repeated the analyses restricting to tonsillectomies indicated strictly due to tonsillitis, the results were similar to those shown in Table 1 (Table S7).

\section{Discussion}

In a cohort of 2.4 million persons, we observed strong and consistent familial aggregation of tonsillectomy. A family history of tonsillectomy was associated with as much as an 11-fold increased risk of tonsillectomy, depending on the number and degree of relatedness of tonsillectomized relatives. The familial aggregation was strongest for children $<10$ years of age. The observed hereditary pattern of associations was compatible with a strong genetic component both in children $<10$ years of age and in those aged 10-19 years. However, there was also a substantial shared environmental component among the younger children that was not evident among children aged 10-19 years.

Our findings, based on almost 150,000 tonsillectomies, add population-based clinical evidence that there is a genetic component to chronic and/or recurrent acute tonsillitis leading to tonsillectomy, and describe age- and sex-specific aspects of this component that have never before been reported. Previously, a Norwegian study of 9479 twins aged 18-25 years provided evidence of a genetic predisposition for self-reported recurrent tonsillitis independent of sex, ${ }^{12}$

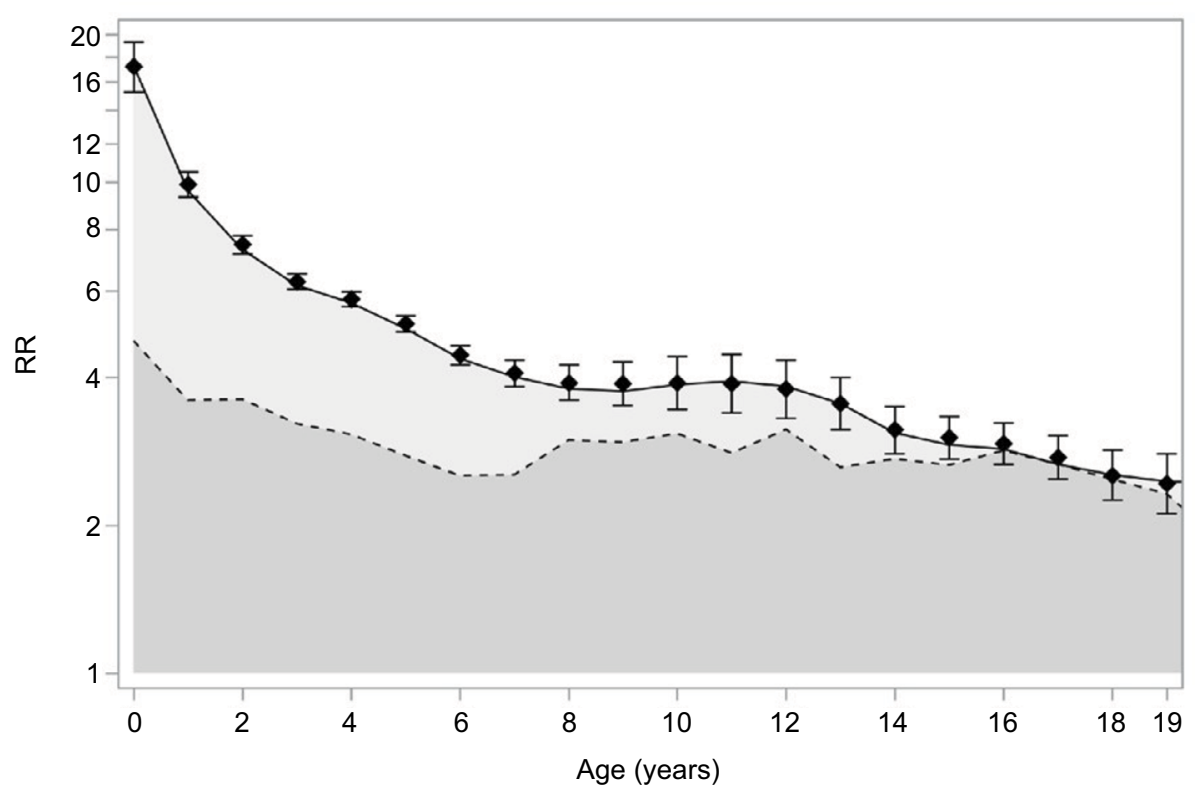

Figure 2 Age-specific RR for tonsillectomy following tonsillectomy in a sibling, Denmark, 1977-20I3; an analysis among individuals with I sibling (i.e., RRs for pairs of siblings). Notes: The points represent the "observed" RRs with $95 \% \mathrm{Cls}$. The solid line represents the estimated total (gray and dark gray) area, while the area under the dotted line (the dark gray area) indicates the genetic component of the estimated RR. The light gray area between the solid and dashed lines indicates the shared environment component of the estimated RR.

Abbreviation: RR, rate ratio. 
and an Australian study of 7620 adult twins hinted at a genetic predisposition for self-reported tonsillectomy. ${ }^{13} \mathrm{On}$ the basis of the same case definition as in the present study, we recently conducted a genome-wide association study (GWAS) of tonsillectomy ( $\sim 3000$ cases) and identified and replicated an association between a genetic variant in the gene HORMAD2 and tonsillectomy. ${ }^{26}$ Another recent GWAS based on self-reported tonsillectomy identified 36 genetic variants associated with tonsillectomy (60 cases), ${ }^{27}$ but did not include replication of these associations in samples with clinically documented tonsillitis and tonsillectomy. Further studies are needed to characterize the functional mechanisms behind the associations.

Observed associations with tonsillectomy in relatives were strongest for children $<10$ years of age. Modeling of the genetic and environmental contributions to these associations suggested that this was due to a stronger contribution of shared environment in this age group than in older children. In contrast, the genetic contribution to the familial aggregation of tonsillectomy appeared to be constant throughout childhood.

Although our genetic model made certain assumptions, estimation of the genetic and environmental components of the familial aggregation of tonsillectomy was based directly on the observed RRs, which agreed well with the expected RRs estimated by the model (Figure 1). Interpretation of the shared environment findings should also occur in the context of the specific model used. First, shared environment includes not only physical proximity and shared behaviors but also a shared threshold for intervention by tonsillectomy, given that families often share a general practitioner, views on the advisability of tonsillectomy vs waiting, and/or a local hospital or clinic (at which the surgery is performed), for example, having a particular professional culture (sometimes referred to as a "surgical signature"). ${ }^{28,29}$ Second, since shared environment will likely be more extensive for persons living together, the shared environment component in the model was allowed to differ for pairs of relatives with and without the same mother (an indicator of shared household). Third, since the first year of risk time after tonsillectomy in a relative was excluded from the analysis, the aspect of shared environment directly related to tonsillectomy per se (i.e., a shared contagion) was, to a large degree removed from the estimation.

Age-specific differences in the environmental contribution to the familial aggregation of tonsillectomy might help to explain the age- and sex-specific differences in tonsillectomy rates that have been noted in most reports from the last 40-50 years..$^{5,8,9,30}$ The general observation has been that tonsillectomy rates peak in early childhood (predomi- nantly in males) and again in adolescence (predominantly in females)..$^{5,8,9}$ It has been speculated that parental preferences to tonsillectomy or different rates of chronic and/ or recurrent acute tonsillitis in males and females might explain these differences. ${ }^{5,9}$ Our analysis provides a possible explanation for these differences, which have previously been difficult to study. We observed no strong contribution from shared familial environment among 10-19 year olds for either males or females; the genetic contribution in this age group corresponded to that for younger children. This finding supports the suggestion that in addition to a genetic contribution, the tonsillectomy peak in this age group could have non-genetic sources that are not predominantly shared among family members (e.g., sources associated with school or friends). In contrast, our findings in younger children support the hypothesis that the peak in tonsillectomy at 4-5 years of age has a strong non-genetic source predominantly shared among family members (e.g., siblings may share a threshold for tonsillectomy intervention by using the same general practitioner and local hospital).

The study was based on mandatory national (nonretrospective) registration of vital status, kinship links and disease in Denmark over 36 years. This unbiased inclusion of about half of the entire Danish population made selection bias negligible and allowed us to accrue a very large number of tonsillectomized individuals, permitting analyses stratified not only by relatedness but also by number of relatives of a given type.

As for other surgical operations in Denmark, ${ }^{31}$ registration of tonsillectomies is close to complete. The incidence was 144 per 100,000 person-years, ranging up to 1000 for the 2 age-specific peaks. ${ }^{5}$ In comparison, smaller studies range from 20 to $1000.6,8,29,30,32,33$ A potential concern when we used tonsillectomy to address genetic control of the tonsillar immune response to infection, was that tonsillectomy is increasingly performed for a less infection-related cause than tonsillitis, notably tonsilar and adenoid hyperthrophy causing obstructive sleep apnea. ${ }^{8,34,35}$ However, we adjusted RRs for calendar year and in the additional analysis, we found similar RRs when excluding tonsillectomies caused by tonsil hypertrophy or performed as adenotonsillectomies.

\section{Conclusion}

Our findings suggest that genetic factors predispose to severe tonsillitis underlying tonsillectomy, regardless of age and sex. Further studies are needed to understand how genes regulate the tonsils' immune response to infections, and for example, whether genetic considerations could benefit the treatment of severe tonsillitis. 


\section{Acknowledgments}

This work was supported by Oak Foundation Fellowships (grant number OCAY-12-319 to Drs Bager and Feenstra).

\section{Disclosure}

The authors report no conflicts of interest in this work.

\section{References}

1. Perry ME. The specialised structure of crypt epithelium in the human palatine tonsil and its functional significance. J Anat. 1994;185(Pt 1): 111-127.

2. Burton MJ, Glasziou PP, Chong LY, Venekamp RP. Tonsillectomy or adenotonsillectomy versus non-surgical treatment for chronic/recurrent acute tonsillitis. Cochrane Database Syst Rev. 2014;11:CD001802.

3. Mitka M. Guideline cites appropriateness criteria for performing tonsillectomy in children. JAMA. 2011;305(7):661-662.

4. Georgalas CC, Tolley NS, Narula PA. Tonsillitis. BMJ Clin Evid. 2014;2014:0503.

5. Vestergaard H, Wohlfahrt J, Westergaard T, Pipper C, Rasmussen N, Melbye M. Incidence of tonsillectomy in Denmark, 1980 to 2001. Pediatr Infect Dis J. 2007;26(12):1117-1121.

6. Van Den Akker EH, Hoes AW, Burton MJ, Schilder AG. Large international differences in (adeno)tonsillectomy rates. Clin Otolaryngol Allied Sci. 2004;29(2):161-164.

7. Koshy E, Murray J, Bottle A, et al. Significantly increasing hospital admissions for acute throat infections among children in England: is this related to tonsillectomy rates? Arch Dis Child. 2012;97(12):1064-1068.

8. Erickson BK, Larson DR, St Sauver JL, Meverden RA, Orvidas LJ. Changes in incidence and indications of tonsillectomy and adenotonsillectomy, 1970-2005. Otolaryngol Head Neck Surg. 2009;140(6):894-901.

9. Freeman JL, Jekel JF, Freeman DH Jr. Changes in age and sex specific tonsillectomy rates: United States, 1970-1977. Am J Public Health. 1982;72(5):488-491.

10. Stephenson J. Studies probe new anti-HIV strategy, long-term success of prevention methods. JAMA. 2011;305(14):1397-1399.

11. Koch A, Melbye M, Sorensen P, et al. Acute respiratory tract infections and mannose-binding lectin insufficiency during early childhood. JAMA. 2001;285(10):1316-1321.

12. Kvestad E, Kvaerner KJ, Roysamb E, Tambs K, Harris JR, Magnus P. Heritability of recurrent tonsillitis. Archiv Otolaryngol Head Neck Surg. 2005;131(5):383-387.

13. Martin NG, Kehren U, Battistutta D, Mathews JD. Iatrogenic influences on the heritability of childhood tonsillectomy: cohort differences in twin concordance. Acta Genet Med Gemellol (Roma). 1991;40(2):165-172.

14. Pedersen CB. The Danish Civil Registration System. Scand J Public Health. 2011;39(7 Suppl):22-25.

15. Pedersen CB, Gotzsche H, Moller JO, Mortensen PB. The Danish Civil Registration System. A cohort of eight million persons. Dan Med Bull. 2006;53(4):441-449.

16. Andersen TF, Madsen M, Jorgensen J, Mellemkjoer L, Olsen JH. The Danish National Hospital Register. A valuable source of data for modern health sciences. Dan Med Bull. 1999;46(3):263-268.

17. Andersen JS, Olivarius NF, Krasnik A. The Danish National Health Service Register. Scand J Public Health. 2011;39(7 Suppl):34-37.

18. Olivarius NF, Hollnagel H, Krasnik A, Pedersen PA, Thorsen H. The Danish National Health Service Register. A tool for primary health care research. Dan Med Bull. 1997;44(4):449-453.
19. Ovesen T, Kamarauskas A, Hlidarsdottir T, Dahl MR, Mainz J. Good long-term results after tonsillectomy in ear, nose and throat practices. Dan Med J. 2013;60(5):A4637.

20. Oyen N, Ranthe MF, Carstensen L, et al. Familial aggregation of lone atrial fibrillation in young persons. $\mathrm{J} \mathrm{Am}$ Coll Cardiol. 2012;60(10): 917-921.

21. Schnack TH, Poulsen G, Myrup C, Wohlfahrt J, Melbye M. Familial coaggregation of cryptorchidism, hypospadias, and testicular germ cell cancer: a nationwide cohort study. J Natl Cancer Inst. 2010;102(3): 187-192.

22. Boyd HA, Poulsen G, Wohlfahrt J, Murray JC, Feenstra B, Melbye M. Maternal contributions to preterm delivery. Am J Epidemiol. 2009;170(11):1358-1364.

23. Rostgaard K, Wohlfahrt J, Hjalgrim H. A genetic basis for infectious mononucleosis: evidence from a family study of hospitalized cases in Denmark. Clin Infect Dis. 2014;58(12):1684-1689.

24. Rostgaard K, Nielsen TR, Wohlfahrt J, et al. Sibship structure and risk of infectious mononucleosis: a population-based cohort study. Int J Epidemiol. 2014;43(5):1607-1614.

25. Wright S. Coefficients of inbreeding and relationship. Am Nat. 1922;56(645):330-338.

26. Feenstra B, Bager P, Liu X, et al. Genome-wide association study identifies variants in HORMAD2 associated with tonsillectomy. J Med Genet. 2017;54(5):358-364.

27. Pickrell JK, Berisa T, Liu JZ, Segurel L, Tung JY, Hinds DA. Detection and interpretation of shared genetic influences on 42 human traits. Nat Genet. 2016;48(7):709-717.

28. Weinstein JN, Bronner KK, Morgan TS, Wennberg JE. Trends and geographic variations in major surgery for degenerative diseases of the hip, knee, and spine. Health Aff (Millwood). 2004;Suppl Variation:VAR81-VAR89.

29. National Health Service PHE. The NHS atlas of variation in Healthcare. 2015 compendium, Map 88: rate of elective admission to hospital for tonsillectomy in children aged 0-17 years per population by Clinical Commission Group (211 areas). National Health Service, Public Health of England; 2015;228-229. Available from: https://fingertips.phe.org. uk/profile/atlas-of-variation. Accessed May 31, 2017.

30. McPherson K, Wennberg JE, Hovind OB, Clifford P. Small-area variations in the use of common surgical procedures: an international comparison of New England, England, and Norway. $N$ Engl J Med. 1982;307(21):1310-1314.

31. Schmidt M, Schmidt SA, Sandegaard JL, Ehrenstein V, Pedersen L, Sorensen HT. The Danish National Patient Registry: a review of content, data quality, and research potential. Clin Epidemiol. 2015;7: 449-490.

32. Bhattacharyya N, Lin HW. Changes and consistencies in the epidemiology of pediatric adenotonsillar surgery, 1996-2006. Otolaryngol Head Neck Surg. 2010;143(5):680-684.

33. Pearson RJ, Smedby B, Berfenstam R, Logan RF, Burgess AM Jr., Peterson OL. Hospital caseloads in Liverpool, New England, and Uppsala. An international comparison. Lancet. 1968;2(7567): 559-566.

34. Marcus CL, Moore RH, Rosen CL, et al. A randomized trial of adenotonsillectomy for childhood sleep apnea. N Engl J Med. 2013;368(25): 2366-2376.

35. Kalampouka E, Moudaki A, Malakasioti G, PanaghiotopoulouGartagani P, Chrousos G, Kaditis AG. Family history of adenotonsillectomy as a risk factor for tonsillar hypertrophy and snoring in childhood. Pediatr Pulmonol. 2014;49(4):366-371. 
Clinical Epidemiology is an international, peer-reviewed, open access, online journal focusing on disease and drug epidemiology, identification of risk factors and screening procedures to develop optimal preventative initiatives and programs. Specific topics include: diagnosis, prognosis, treatment, screening, prevention, risk factor modification,

Submit your manuscript here: https://www.dovepress.com/clinical-epidemiology-journal
Dovepress

systematic reviews, risk and safety of medical interventions, epidemiology and biostatistical methods, and evaluation of guidelines, translational medicine, health policies and economic evaluations. The manuscript management system is completely online and includes a very quick and fair peer-review system, which is all easy to use. 